\title{
TWISTER MATEMÁTICO: UNA MANERA PRÁCTICA DE ENSEÑAR Y APRENDER A PARTIR DEL JUEGO
}

\author{
Fabián Leonardo González Rincón \\ Licenciado en Matemáticas \\ Universidad Pedagógica y Tecnológica de Colombia \\ Unipanamericana /Fundación Universitaria Panamericana \\ flgonzalezr@unipanamericana.edu.co
}

\author{
Gigliola Andrea Rojas Muñoz \\ Licenciada en Educación Preescolar \\ Universidad Pedagógica y Tecnológica de Colombia \\ giglis17@hotmail.com
}

Cómo citar este artículo

González, F. y Rojas, G. (2013) Twister matemático: una manera práctica de enseñar y aprender a partir del juego. Espiral, Revista de Docencia e investigación. 3 (2)

\section{Resumen}

En el año 2010 surge un proyecto de investigación que ha pretendido colaborar con los procesos de enseñanza aprendizaje de la matemática en la educación preescolar brindando ideas que contribuyan e incentiven a trabajar con actividades escolares basadas en el juego. En este documento se plasma una reflexión relacionada con el Proyecto denominado El Twister Matemático el cual obtuvo una nota laureada por su innovación pedagógica. El Twister Matemático busca innovar los procesos de enseñanza aprendizaje vinculando los procesos básicos de pensamiento y los procesos lógico-matemáticos en los niños y las niñas del nivel de transición, llevándolos a la adquisición de capacidades o habilidades cognitivas fundamentales para el proceso formativo del niño de edad preescolar. Dicho proyecto tiene un método de investigación cualitativo, a través de la Investigación Acción debido a que presenta resultados descriptivos y específicos que van acorde a la temática que se trabajó. En la misma medida, se pudo observar la realidad de la comunidad objeto de estudio teniendo en cuenta sus formas de pensar, sentir y actuar frente a las estrategias propuestas; la modificación del Twister ${ }^{\mathrm{TM}}$ benefició en gran medida a la comunidad objeto de estudio, además con este tipo de investigación se pudieron observar, corregir y suplir las necesidades exploradas proporcionando alternativas de solución que favorecieron la implementación de la propuesta, ampliamente acogida por el contexto en el que se aplicó.

Palabras clave: educación preescolar, juego, procesos básicos de pensamiento (P.B.P.), nociones lógico-matemáticas, Twister ${ }^{\mathrm{TM}}$.

\begin{abstract}
In 2010 a research project was conducted to collaborate with teaching - learning of mathematics in preschool education by providing ideas that help and encourage working with school activities based on the game. A reflection about the project entitled the Mathematical Twister is introduced in this paper, which received a laureate qualification due to its innovative teaching strategy. The Mathematical Twister is intended to find an innovating way of teaching and learning processes. This idea attempts to link the basic processes of thought and logicalmathematical processes in children of transition degree in order to take them to acquire basic cognitive abilities or skills. This project has a qualitative research method, specifically "action research" because it presents descriptive and specific results, according to the theme to be worked. To the same extent, reality of the community was perceived under observation of their ways of thinking, feeling and acting through proposed strategies. Twister $^{\mathrm{TM}}$ modification greatly benefited the community under study, and with this type of research, some needs of the community were observed, corrected and met by providing alternative solutions that contributed to the implementation of this proposal, which was widely welcome by the context in which it was applied.
\end{abstract}

Keywords: preschool Education, game, basic thought processes, logical-mathematical notions, Twister game ${ }^{\mathrm{TM}}$.

\section{Introducción}

Uno de los grandes retos de la educación preescolar es optimizar el proceso de formación y desarrollo de los niños y niñas de 0 a 6 años, 


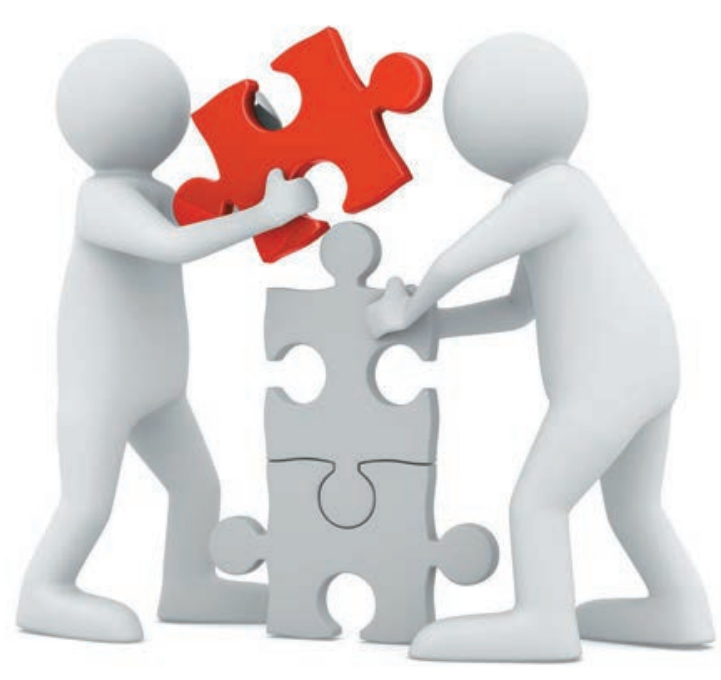

procurando que los educadores sean efectivos mediadores entre el mundo y los niños y niñas. El docente-mediador debe promover su desarrollo, no detenerlo ni entorpecerlo, para lo cual, propone, pero no impone; exige pero no satura, es firme pero no agresivo; está presente cuando el niño lo necesita y se aleja cuando su presencia lo inhibe.

Según De Murzi (2004) las acciones educativas, en los primeros años de vida, deben estar dirigidas a estimular el desarrollo cognitivo, emocional, de lenguaje, físico, motor, social, moral y sexual de los niños y niñas, de tal manera que no perdamos el espacio más relevante en la vida del ser humano para desarrollar sus potencialidades, muy especialmente para promover su inteligencia.

Es por esta y otras razones que en el año 2010 surge la idea de diseñar un proyecto que responde a los retos de la educación infantil, a partir de un diagnóstico realizado en la Institución Educativa Julius Sieber, localizada en un contexto urbano, en el barrio Asís (Calle 64A No 9-25), en la Ciudad de Tunja, en el departamento de Boyacá. Dicho diagnóstico se obtiene a partir de la observación directa dentro del aula de clase, una entrevista con la docente del grado transición $\mathrm{C}$ y una encuesta realizada a padres de familia, actividades evidenciadas en un diario de campo y un proceso de sistematización de las mismas, sin llegar a interferir en las actividades de formación normales de los niños(as); desde este proceso se observaron ciertos aspectos que motivaron la creación e implementación de un proyecto que respondiera a las necesidades de los niños y niñas del grado transición $\mathrm{C}$ de dicha institución.

Una de las falencias encontradas en este grado, es el escaso material didáctico y audiovisual para el uso de los niños y las niñas, además de un tiempo reducido para las actividades lúdicas, una gran implementación de hojas base que muchas veces terminan por cansar al infante y desmotivarlo y un afán de la maestra por no perder el ritmo de sus compañeras de la institución (ya que desafortunadamente, los padres de familia miden más la cantidad de lo trabajado que la calidad de esto mismo). Estos factores generan una competencia entre el afán de cumplir metas factibles para los encargados de la educación infantil en general y el aspecto cognitivo de los niños y las niñas, donde el gran triunfador suele ser el afán de entregar la carpeta con más hojas a fin de año.

\section{Metodología}

Uno de los enfoques de este proceso investigativo es el aprendizaje lógico-matemático, además de un fortalecimiento de las Habilidades Básicas de Pensamiento (HBP), sin dejar de lado otras áreas del conocimiento, integrando conceptos e ideas previas y apoyando la labor educativa de la docente preescolar, para una mejor comprensión de temas tratados dentro del aula. Cabe resaltar que en este tiempo a pesar de los avances investigativos en educación y de los recursos que actualmente brinda la tecnología, además del material de apoyo encontrado en medios virtuales, en las aulas es común ver que la educación tradicional reina, sin ser siempre culpa del maestro sino muchas veces los escasos recursos destinados para las 
escuelas y aunque el maestro debería preocuparse por la elaboración del material, el tiempo y los recursos son mínimos e impiden la construcción de este mismo.

Las HBP como herramientas importantes en el desarrollo cognitivo del niño, son aquellas que para Guevara "sirven para sobrevivir en el mundo cotidiano, tienen una función social y visto de esta manera es importante que el estudiante no las haga a un lado". (2000, pág. 44). Lo que debe quedar claro es que no son suficientes para sobrevivir en la escuela, son una herramienta pero no, lo más importante para conseguir el nivel cognitivo deseado. Dichas habilidades se integran en el proyecto y de la mano con el juego, hacen del aprendizaje lógico matemático una actividad placentera y dinámica para la obtención de resultados favorables, no sin antes formular la pregunta: ¿De qué manera el Twister Matemático como estrategia lúdicopedagógica fortalece las habilidades cognitivas para el desarrollo de algunas nociones lógicomatemáticas en los niños y las niñas de transición $\mathrm{C}$ de la institución educativa Julius Sieber?

Es por esta razón, que los investigadores formulan un proyecto lúdico-pedagógico que intenta dar respuesta a dicho interrogante y además contribuya con las necesidades educativas de los niños y las niñas a través del clásico juego Twister ${ }^{\mathrm{TM}}$, el cual al ser modificado, ofrece una forma diferente de enseñar matemáticas y a la vez trabajar la corporalidad del niño(a) junto con el aspecto cognitivo, integrando otras áreas de conocimiento y a la vez trabajando las HBP importantes en el transcurso de la vida del niño(a) por la escuela.

El proyecto Twister matemático comienza como un intento por mostrar la importancia del uso de juegos tradicionales en el aula y el gran aprovechamiento que estos brindan en la producción de resultados en la cognición del niño(a), además de una unión inseparable entre el juego y el aprendizaje matemático, Sierra \& Guédez (2006) afirman que no es lo usual que el niño juegue para aprender matemáticas, pero el contenido de un juego apropiado es el medio para motivar el aprendizaje de nuevos conocimientos y habilidades, que serán sistematizados y mejor estructurados para avanzar en el proceso de abstracción lógico-matemático y crear unidades de conocimientos cada vez más complejos.

Los juegos matemáticos, cuando son formulados y reglamentados con claridad, pueden convertirse en herramientas valiosas para suavizar el camino del niño(a) en la elaboración de generalizaciones, el aumento de su capacidad de retentiva y de aplicación de los conceptos matemáticos en el mundo que lo rodea (Sierra \& Guédez, 2006).

Es importante aclarar que la matemática no es un juego en sí y que tampoco está ligada al juego, pero éste se convierte en una estrategia para motivar dicho aprendizaje. El reto es, entonces, descubrir o construir actividades que sean realmente juegos para los niños y que, a la vez, propicien aprendizajes matemáticos interesantes y significativos; y el Twister ${ }^{\mathrm{TM}}$ a nuestro modo de ver no está desligado del aprendizaje matemático ni del proceso cognitivo que ampara al juego, el cual es además un fuerte impulsor de vínculos afectivos, de autoestima, del desarrollo de la corporalidad y además un gran influyente en el manejo de reglas y en la aceptación del triunfo y la derrota, tomando a ésta última como una oportunidad para seguir avanzando en los objetivos propuestos.

Piaget (1969) dice que las matemáticas son, antes que nada y de manera más importante, acciones ejercidas sobre cosas, y las operaciones por sí mismas son acciones e interpreta que todos los niños evolucionan a través de estadios. La teoría de Piaget descubre los estadios de desarrollo cognitivo desde la infancia a la adolescencia: cómo las estructuras psicológicas se desarrollan a partir de los reflejos innatos, se organizan durante la infancia en esquemas de conducta, se internalizan durante el segundo 
año de vida como modelos de pensamiento, y se desarrollan durante la infancia y la adolescencia en complejas estructuras intelectuales que caracterizan la vida adulta, dichos estadios son tenidos en cuenta en este proyecto, partiendo de la idea de que los niños del grado transición $\mathrm{C}$ son niños que se encuentran en la etapa preoperacional (etapa del pensamiento y del lenguaje que gradúa su capacidad de pensar simbólicamente (Piaget, 1969)) adoptando la teoría de Piaget para el planteamiento de actividades apropiadas en dicha etapa.

Piaget brinda la teoría apropiada para la ejecución de la propuesta, en la cual se evidencian conductas de los niños que se relacionan con la etapa preoperacional, ya que los pequeños, objeto de estudio, son capaces de organizar el espacio situando, desplazando los objetos (dentro/fuera, encima/debajo, delante/detrás, arriba/abajo), conceptos y vocabularios básicos, tienen la capacidad de descubrir propiedades físicas de los objetos que manipulan: longitud, distancia, cantidad, mezcladas con las cualidades perceptivas y además comparan objetos en función de cualidades físicas, condiciones importantes en el desarrollo de su parte cognitiva.

Por otra parte, el desarrollo de las HBP se inserta con el fin de despertar en el niño procesos de pensamiento que contribuyan al desarrollo del aprendizaje y a una mejor comprensión de los temas, dichas habilidades sirven para transitar en el mundo cotidiano, por eso es importante que se reconozcan para que se sepan utilizar, tienen una función social y no son suficientes para el estudiante en su tránsito por la escuela. Lo que se pretende es que, al reconocer estas habilidades, las apliquen y hagan transferencia de ellas de manera consciente, ya que les proporcionarán la experiencia de comprender de manera general cualquier situación o tema (Cruz, 1999). De lo que se trata es, que el estudiante se dé cuenta que las utiliza de manera irreflexiva, y que al reconocerlas las use de manera contextualizada. Este proceso implica que adopten ciertas actitudes que favorecen su desarrollo, tales como apertura, disposición a la práctica y curiosidad, (Piaget, 1969) entre otras.

Es importante aclarar que aunque el juego fue uno de los grandes componentes, no hubiera sido lo mismo sin la ayuda del cuento, ya que basados en Dienes (1977) se construyeron las acciones investigativas de acuerdo a las etapas que plantea para el aprendizaje de las matemáticas. Dienes (1977) da cierta jerarquía al juego como factor fundamental en la comprensión del tema, además de la gran importancia que le da al juego libre como introductor en el desarrollo de este mismo, insertando a la vez actividades de construcción (donde se evidencia el Twister $^{\mathrm{TM}}$ como estrategia), representación gráfica y actividades de confrontación haciendo de la investigación una labor provechosa con un orden lógico y coherente, respondiendo a las temáticas tratadas.

Es de resaltar que este proyecto se trabajó de manera interdisciplinar entre la Licenciatura en matemáticas y la Licenciatura en Educación Preescolar, así, se creó una retroalimentación de conocimientos, y se demostró las posibilidades de trabajar entre licenciaturas, generando puentes de comunicación que contribuyen al desarrollo de proyectos como éste a futuro.

La estrategia lúdico-pedagógica el Twistermatemático se desarrolla en 10 talleres lúdicos, que contienen algunas nociones de espacio como adelante-atrás, derecha izquierda, horizontal-vertical y otras lógico-matemáticas como la clasificación, la seriación y la correspondencia. Dichas acciones van de la mano con las HBP, todas trabajadas a través de modificaciones del clásico juego Twister ${ }^{\mathrm{TM}}$ integrando otras áreas de conocimiento a partir de la interacción con diferentes materiales, dando variadas posibilidades para que el niño(a) explore, conozca, interactúe, se divierta y a la vez aprenda las matemáticas de una manera diferente e interactiva, vinculando a las figuras geométricas como parte fundamental en 
el desarrollo del proyecto y permitiendo al niño(a) la oportunidad de brindar soluciones en el planteamiento de problemas para un mejor desarrollo cognitivo, socio-afectivo y corporal.

\section{Resultados}

Estos talleres son de interés para los niños(as) ya que lo insertan en un mundo diferente, con personajes e historias llenas de fantasía. Además, les permite vivenciar cada una de las situaciones y los motiva a través del juego, especialmente del Twister ${ }^{\mathrm{TM}}$, el cual los deja interactuar con su cuerpo y el de sus compañeros. También genera en los niños(as) una disposición acorde a las expectativas planteadas, observándose interés por la narración del cuento. Asimismo de un amplio desarrollo de la participación y el trabajo en equipo; es esencial también resaltar la labor del juego libre, el cual posee cierta importancia de este mismo en el manejo de las temáticas, ya que el niño(a) sin imaginarlo se introduce en cada tema, entonces al llegar al momento de la explicación ya existe un conocimiento previo, trabajado a través de la espontaneidad que brinda el juego libre en este tipo de investigaciones.

Durante el desarrollo del proyecto se evaluaron por medio de sistematizaciones, los procesos de aprendizaje en cuanto a las nociones lógico-matemáticas y los procesos básicos de pensamiento, teniendo en cuenta las dificultades observadas y planteando en cada una soluciones prontas y apropiadas que fortalecieron su aprendizaje. Por medio de estas evaluaciones se valoró el avance y/o el progreso tanto de los niños como del planteamiento de cada uno de los talleres; se aplicaron 10 talleres investigativos acorde a las necesidades de la población, incluyendo de esta manera juegos y representaciones de cuentos que dieron la posibilidad al niño(a) de vivenciar situaciones problema dentro del desarrollo de los talleres, permitiendo la comprensión de cada una de las temáticas.

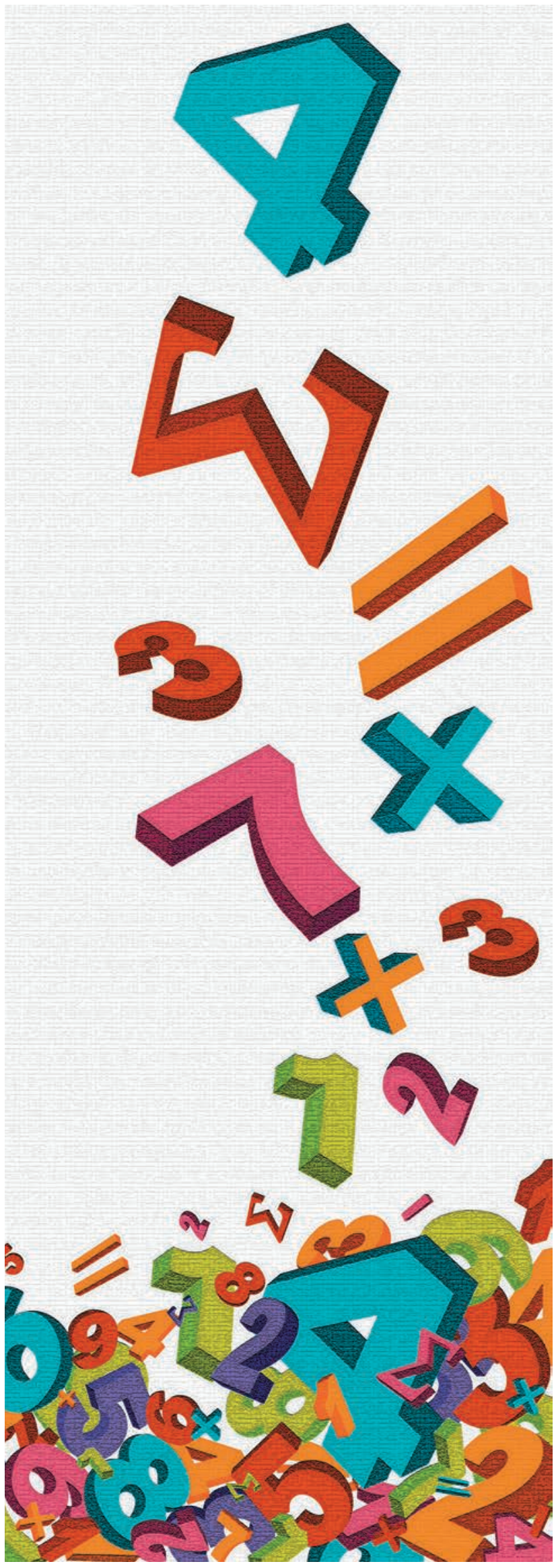




\section{Conclusiones}

En general se observa un proceso formativo importante, aunque cabe resaltar que un porcentaje bajo de niños(as) no siempre comprende la totalidad de las temáticas, especialmente la seriación, la cual presenta inconvenientes debido a que algunos de ellos tienen dificultades al momento de descubrir secuencias, principalmente al trabajar con grosor, posición y forma. En dichas temáticas es común encontrar distracción por parte de los pequeños, pero al final la mayoría de los niños demuestran su habilidad innata en la solución de problemas.

En cuanto a las demás temáticas cabe resaltar que en su mayoría los niños(as) responden positivamente, ya que a través del juego ejercitan sus procesos cognitivos y el desarrollo de las HBP, argumentando, analizando interpretando y planteando soluciones ante diferentes situaciones problemas presentadas en el desarrollo las acciones investigativas. Después del desarrollo de este tipo de proyectos es común ver que los niños formulan y resuelven preguntas de manera más fluida y segura, y se ve un poco más de conciencia frente a sus HBP, las cuales sigue en su orden lógico, fortaleciendo sus procesos de pensamiento, y sacando de

\section{Twister Matemático}

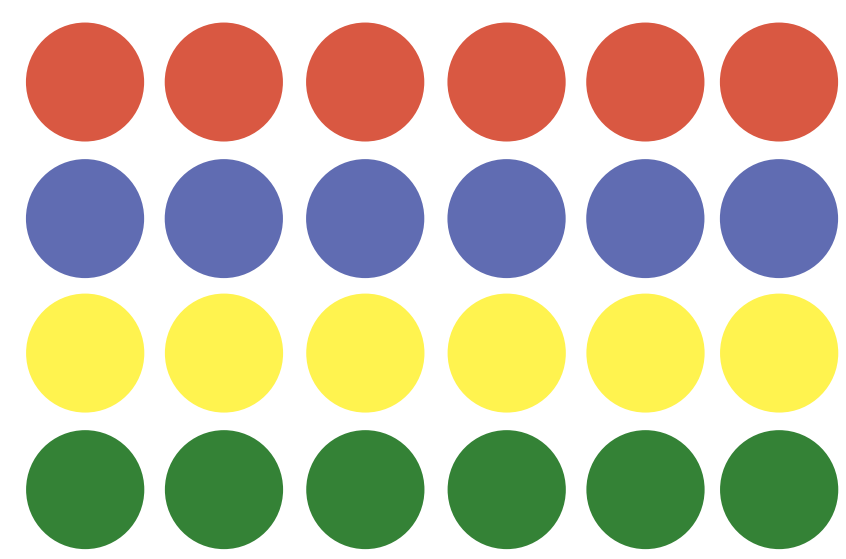

adentro a este individuo inquieto por aprender y con un pensamiento crítico y formativo para su proceso de desarrollo.

Vale la pena anotar que este tipo de acciones innovadoras son posibles gracias a la actitud activa del docente, que no interfiere en el pensamiento del niño(a), quien no condiciona y da al infante recursos pero no soluciones, creando de esta manera una independencia activa en cada uno de sus estudiantes, incentivando siempre el deseo por aprender y ese interés por no flaquear ante los inconvenientes, sino verlos como una oportunidad para seguir luchando por alcanzar metas aún más altas que las propuestas; siempre ofreciendo propuestas pertinentes, teniendo en cuenta la edad, valiéndose de diagnósticos que aporten ideas y opciones de investigación, que contribuyan a la formación de los seres del mañana, generando ambientes favorables para la obtención de resultados y favoreciendo al óptimo desarrollo del pensamiento sin dejar de lado los valores y las buenas costumbres.

\section{Referencias}

Cruz, G. E. (1999). Habilidades Básicas de Pensamiento: Nivel Prerreflexivo de COL. En A. Campirán, G. Guevara, \& L. Sánchez , Habilidades de Pensamiento Crítico y Creativo (pág. 67). Veracruz, Xalapa, México: Colección Hiper-COL.

De Murzi, F. E. (2004). La Educación Preescolar un derecho que tiene la infancia a participar en situaciones educativas que sirvan para impulsar su desarrollo integral. Acción pedagógica, 13(2), 136 - 140.

Dienes, Z. P. (1977). Las Seis etapas del Aprendizaje en Matemáticas. Barcelona: Teide.

Guevara, G. (2000). Habilidades Básicas [Paráfrasis], Manuscrito no Publicado. México: Facultad de Filosofía, U.V.

Piaget, J. (1969). Psicilogía del Niño. Madrid: Ediciones Morata.

Sierra, D., y Guédez, C. (2006). Juego y Aprendo a Calcular. Venezuela: Fe y Alegría. 